\title{
Intervención artística para la mejora psicosocial de un caso de Necesidades Educativas Especiales
}

\author{
Laura Martín Martínez. Universidad de Granada \\ Pedro Chacón-Gordillo. Universidad de Granada
}

Recepción: 6.02.2018 | Aceptado: 12.02.2018

Correspondencia a través de ORCID: Pedro Chacón Gordillo

0000-0002-0720-0115

Citar: Martin, L. y Chacon-Gordillo, P. (2018). Intervención artística para la mejora psicosocial de un caso de Necesidades Educativas Especiales. ReiDoCrea, 7, 17-27.

\begin{abstract}
Resumen: Tanto la expresión artística como corporal resultan fundamentales en el proceso de desarrollo del niño/a. El objetivo de esta investigación se basa en reducir la conducta disruptiva de un menor que es incluido en el programa de Necesidades Educativas Especiales (NEE) mediante una intervención de dramatización. Método: estudio experimental prospectivo de caso único realizado en el transcurso de cuatro meses en un centro de atención terapéutica de la Comunidad de Madrid. En la intervención participaron nueve menores, el caso objeto de estudio fue seleccionado entre los participantes de la intervención de dramatización mediante muestreo por conveniencia (Ochoa, 2015). La evaluación del caso se desarrolló durante 4 meses en los que se realizaron 30 sesiones de dramatización, utilizando los siguientes instrumentos para cumplir el objetivo: muestreo por conveniencia (McMillan \& Schumacher, 2001) y registros de observación en los que se cuantificaron las conductas disruptivas del caso. Resultados: Los registros tomados durante las 30 sesiones de dramatización muestran una notable disminución en el número de conductas disruptivas del caso. El cuestionario de preguntas abiertas destinado a la familia del caso reúne información que registran cambios significativos en la conducta del caso tras la intervención de dramatización. Conclusiones: La dramatización como recurso terapéutico resulta eficaz para la mejora psicosocial de menores que presentan un comportamiento disruptivo.
\end{abstract}

Palabras clave: Educación | Dramaterapia

\section{Artistic intervention for the psychosocial improvement of a case of Special Educational Needs}

Abstract: Both artistic and physical expression are fundamental in the development process of the child. The objective of this research is based on reducing the disruptive behavior of a minor in the Special Educational Needs (SEN) program through a dramatization intervention. Method: A prospective experimental study of a single case was carried out over the course of four months in a therapeutic care center of the Community of Madrid. The intervention involved nine children and the case for the study was selected from among the participants of the dramatization intervention by convenience sampling (Ochoa, 2015). The evaluation of the case was carried out over 4 months during which 30 dramatization sessions were conducted, using the following instruments to fulfill the objective: convenience sampling (McMillan \& Schumacher, 2001) and observation records in which the disruptive behaviors of the subject were quantified. Results: The information recorded during the 30 dramatization sessions shows a considerable reduction in disruptive behavior. The questionnaire of open questions addressed to the family of the subject gathers information that registers significant changes in the behavior of the subject after the dramatization intervention. Conclusions: Dramatization as a therapeutic resource is effective for the psychosocial improvement of children with disruptive behavior.

Keywords: Education | Drama Therapy

\section{Introducción}

El sistema educativo debe ofrecer los "medios necesarios para que los alumnos con necesidades educativas especiales, temporales o permanentes, puedan alcanzar, dentro del mismo sistema, los objetivos establecidos con carácter general para todos Ios alumnos." (LOGSE, Título Primero, capítulo II, artículo 36-1). La escuela debe ofrecer adaptaciones curriculares, apoyos específicos o cualquier otra metodología adaptándose a las necesidades que presente el alumnado y ofreciéndole la posibilidad de cubrir sus necesidades educativas. La escuela debe comprometerse a que tanto su 
alumnado de NEE como el resto de alumnos alcancen su desarrollo educativo, social y emocional de la manera más satisfactoria y debe perseguir los mismos objetivos para todos sus alumnos/as.

La UNESCO (2011) determina que las Necesidades Educativas Especiales (NEE) engloban tanto discapacidades físicas, sensoriales, mentales y cognitivas como dificultades de aprendizaje, emocionales y de conducta. A lo largo de la infancia es común en los niños actitudes de desobediencia en determinadas situaciones, así como de negación o en las que mantienen una actitud desafiante ante las personas que representan la autoridad, poniendo a prueba los límites del buen comportamiento. Es lo que conocemos como comportamiento disruptivo (DSM-IV, 1994).

Cuando estos desafíos pasan a ser frecuentes persistiendo en el tiempo, en edades incluso ya avanzadas, puede llegar a convertirse en un problema, tanto presente como en un futuro con diversos trastornos asociados. En casos ya más extremos puede considerarse como un Trastorno Negativista Desafiante (TOD), trastorno que se estima padecen entre el 6 y el 15\% de los niños/as (Alvarez \& Ollendick, 2003). Este trastorno puede ir asociado a otros trastornos como el trastorno de aprendizaje, altos niveles de ansiedad, hiperactividad (TDAH), déficit de atención o trastornos de ánimo. Se caracteriza por presentar conductas negativas, hostiles, de oposición y desafío a la autoridad. Estas conductas se presentan generalmente en forma de enfados, ataque de cólera, desafío a las normas, bajo nivel académico o discusiones con los adultos, puede tratarse de niños/as rencorosos, o que culpabilizan a las personas cercanas. Suelen darse con más frecuencia en el ámbito familiar, pero también en el ámbito escolar o en las relaciones de los niños/as con sus iguales, acentuándose en situaciones que les incomodan (CIE 10, 1994).

Estos síntomas, como se ha comentado, son comunes en los niños/as y se repiten de forma más o menos frecuente según el caso, disminuyendo con el tiempo su frecuencia y terminando por convertirse en hechos aislados. La continuidad en el tiempo es lo que diferencia entre un comportamiento normal, uno disruptivo o va más allá tratándose de un trastorno.

A pesar de que las causas exactas se desconocen existen estudios acerca del posible origen. Una posible causa, según estas teorías sería el aprendizaje, refiriéndose a como las personas del entorno del niño/a han empleado conductas negativas en su educación, o no han sabido ponerse los límites correctamente. Estas actitudes provocan conductas hostiles, desafiantes y negativas en el niño/a ante la autoridad. Otras teorías hablan de problemas surgidos por el excesivo apego con determinados familiares lo que provoca dificultades en la adquisición de la autonomía. O por el contrario, situaciones de violencia o de excesiva permisividad. Situaciones de estrés o ansiedad en los diferentes entornos del niño/a pueden desembocar en un comportamiento disruptivo, generando en el niño/a hostilidad o negación hacia aquello que le provoca ese estado de ansiedad o estrés (Rigau-Ratera, García-Nonell y Artigas-Pallarés, 2006).

Para estos casos son recomendables intervenciones de psicología que reduzcan el nivel de estrés o ansiedad. Estas intervenciones son aconsejables no solo para el niño/a, sino a padres o profesores, y que de esta forma sean capaces de afrontar el problema de forma positiva. No corregir la conducta a tiempo, aparte de los problemas ya inherentes, puede desembocar en problemas en el futuro como conductas asociales, ansiedad, fracaso en la escuela, entre otros (Armas, 2007). 
Teniendo en cuenta lo expuesto, el sistema educativo tiene la responsabilidad de asegurar el bienestar psíquico, físico y emocional de su alumnado y de ofrecer los recursos necesarios que garanticen dicho bienestar. La dramatización como alternativa terapéutica ofrece diferentes recursos para alcanzar la mejora el estado psicosocial de alumnos que son incluidos en los programas de Necesidades Educativas Especiales por presentar trastornos emocionales o de conducta.

La actuación dramática es un estado interior intermedio, un intermediario entre la fantasía y la realidad. La modalidad es ficticia pero la experiencia es muy real. La modalidad ficticia nos permite hacer cosas que aún se encuentran afuera de nuestro alcance en la vida real, tales como expresar emociones temidas, cambiar patrones de conducta o exhibir nuevos rasgos. Una vez que las hemos vivenciado, aunque en modo ficticio, estas nuevas experiencias pueden formar parte del repertorio de nuestra vida real. (Emunah, 1994, p. 27).

Los tratamientos clásicos de psicoterapia hacen uso fundamentalmente del lenguaje verbal como vía de expresión y liberación de emociones. La dramatización ofrece nuevas vías de comunicación que van más allá de lo verbal utilizando la expresión corporal como canal liberador de las emociones. La dramatización le ofrece al niño/a la oportunidad de lidiar con sus dificultades a través del juego y de manera inconsciente. En ocasiones, los niños/as no tienen conciencia de sus comportamientos pero a través de los juegos de dramatización puede hacerlos conscientes. Cuando el niño/a se encuentra inmerso en los juegos de dramatización, vivencia a través de la emoción su propia realidad, experimentando estados de placer y displacer que se van registrando en el inconsciente antes de que se manifiesten de forma consciente (Lapierre y Aucouturier, 1980). Estos autores exponen como "los niños/as deben tener tiempo suficiente para vivir a su ritmo un ciclo fantasmático completo... Es necesario que esos fantasmas sean vivenciados y aceptados hasta el agotamiento del interés del niño" (p. 15). Lapierre y Aucouturier (1980) explican como cuando los intereses fantasmáticos que dominan al niño/a desaparecen, el niño/a se revela como un sujeto receptivo. Cuando el niño/a dramatiza, se transforma en otro sujeto, esta transformación le permite ir alcanzando un desprendimiento de su propio cuerpo que le permite contemplarse como espectador. Como es sabido, el niño/a logra estructurarse subjetivamente cuando su imagen es reflejada en espejo y la discrimina de la imagen del otro Lacan (1946). La propia acción dramática se torna en espejo de su propia realidad que le permite revivirla para reeducarse.

En el año 343 a. C Aristóteles (1990) declaraba que se producía una catarsis en los teatros griegos al presenciar representaciones teatrales que sanaban a los pacientes y restablecía desequilibrios emocionales e incluso curaba enfermedades del alma. Moreno (1946) describe el psicodrama como "una nueva forma de psicoterapia que puede ser ampliamente aplicada" (p.177). Este autor declara que "históricamente el psicodrama representa el punto decisivo en el paso del tratamiento del individuo aislado hacia el tratamiento del individuo en grupos, del tratamiento del individuo con métodos verbales hacia el tratamiento con métodos de acción" (p.10). Moreno (1964) explica como el teatro despliega diferentes acciones terapéuticas en las que el paciente reconstruye una percepción e imagen de sí mismo. Declara como trabajar terapéuticamente mediante intervenciones teatrales significa fomentar la autoafirmación, la autoestima y principalmente la comunicación. Cuando el paciente actúa tiene la oportunidad de expresarse libremente para convertirse en alguien que no es para transformarse en otra persona. La actuación dramática que interpreta el paciente es ficticia pero la experiencia que vivencia en la representación es real ya que modifica su estado emocional dando lugar a nuevas conexiones neuronales. 
La dramatización activa nuevos estados sensorio-emocionales en los que se fusiona el cuerpo, el espacio y la emoción. Cuando el niño/a dramatiza su mundo, va simbolizando de forma reveladora su realidad, su propia escenificación le devuelve imágenes que construyen nuevos pensamientos, mientras actúa tiene la oportunidad dominar su cuerpo y articular su realidad.

El psicodrama es un método de psicoterapia en el cual los pacientes actúan los acontecimientos relevantes de su vida en vez de simplemente hablar sobre ellos. Esto implica explorar en la acción, no sólo los acontecimientos históricos, sino, lo que es más importante, las dimensiones de los acontecimientos psicológicos no abordados habitualmente en las representaciones dramáticas convencionales: los pensamientos no verbalizados, los encuentros con quienes no están presentes, representaciones de fantasías sobre lo que los otros pueden estar sintiendo o pensando, un futuro posible imaginado y muchos otros aspectos de los fenómenos de la experiencia humana.

Aunque el psicodrama es usado habitualmente en un contexto grupal y puede ser un método muy útil para catalizar el proceso grupal ( $\mathrm{y}$, a su vez, ser catalizado por la dinámica grupal), no debe ser considerado como una forma de terapia específicamente grupal. Puede ser usado, como sucede en Francia, con varios co-terapeutas entrenados y un solo paciente. También puede usarse el psicodrama con familias 0 , inclusive, en una forma modificada, en terapias individuales. (Blatner, 1988, p.1)

\section{Objetivo}

Partiendo de la hipótesis de que el uso de la dramatización como recurso terapéutico mejora el estado psicosocial de menores incluidos en los programas de Necesidades Educativas Especiales, se planteó como objetivo de este estudio reducir la conducta disruptiva del caso objeto de estudio disminuyendo su nivel de estrés y ansiedad mediante la intervención del arte. Nos propusimos conseguir dicha mejora en su comportamiento en general y más en concreto en una disminución de su número de enfados, discusiones con adultos, desafío de las reglas, culpas, reproches o mentiras, en definitiva, mejorar el estado de negatividad y desafío en el que se encontraba el caso.

\section{Método}

\section{Participante}

Este estudio experimental prospectivo de caso único se centró en el caso de un menor de 6 años, escolarizado en un centro de la Comunidad de Madrid en el tercer curso de Educación Primaria. Tiene un dictamen de necesidad de atención temprana de psicoterapia, logopedia y psicomotricidad según dictamen técnico facultativo del equipo interdisciplinar de valoración infantil del Centro Regional de Coordinación y Valoración Infantil de la Comunidad de Madrid, por lo que es incluido en el programa de Necesidades Educativas Especiales.

Recibe atención terapéutica en un centro externo de la Comunidad de Madrid, dónde se realiza la intervención de dramatización.

El caso es seleccionado entre varios de los participantes de la intervención de dramatización mediante muestreo por conveniencia (McMillan, \& Schumacher, 2001). En la primera entrevista realizada antes del comienzo de la intervención se considera que es un caso idóneo para el objeto de este estudio. Encaja en el estudio al tratarse de un caso con problemas disruptivos, por estar incluido en el programa de 
Necesidades Educativas Especiales y por encontrarse en un rango de edad entre 5-9 años.

En la entrevista con los padres nos describieron como el caso entre dos y tres presentó las primeras rabietas y como seguían apareciendo con frecuencia, principalmente en el ámbito familiar, cuando se sentía obligado a seguir una norma o no conseguía lo que quería. Destacaron su rigidez y sus dificultades para aceptar la existencia de propuestas diferentes a la suyas. Señalaron sus serias dificultades para expresar sus emociones y en ocasiones para entender las emociones de los demás. Expusieron como mostraba dificultad a la hora de expresar verbalmente emociones, sobretodo el enfado. Destacaron su fácil frustración y su oposición a recibir ayuda del adulto. A través de la entrevista mantenida con los padres, pudimos recopilar información que revelaba como el caso presentaba un pobre control emocional, con tendencia a mostrar oposición ante pequeñas exigencias, principalmente en el ámbito familiar, llegando a mostrar conductas bastante disruptivas como chillar, pegar, tirar las cosas al suelo...

\title{
Diseño
}

El método utilizado en esta investigación fue un estudio experimental prospectivo de caso único. Stake (1985) define el estudio de caso de la siguiente manera:

\begin{abstract}
"Definido como el estudio de un caso sencillo o de un determinado sistema, observa de un modo naturalista e interpreta las interrelaciones de orden superior en el interior de los datos observados. Los resultados son generalizables en lo que a la información dada permite a los lectores decidir si el caso es similar al suyo. El estudio de caso pude y debe ser riguroso. Mientras que el diseño experimental edifica su validez en el interior de su propia metodología, el estudio de casos descansa sobre la responsabilidad del investigador. Si bien otros estilos de investigación buscan elicitar relaciones generales, el estudio de casos explora el contexto de las instancias individuales" (p.277).
\end{abstract}

Esta investigación implicó la utilización y recogida de la experiencia obtenida en el estudio del caso único, observaciones, informes, que describieron mejoras tras la intervención de dramatización. Este estudio se proyectó desde una perspectiva realista con la que se pretendieron producir conocimientos y acciones útiles para que las personas se apoderen o capaciten a través del proceso de construcción y utilización de lo aportado en este estudio. Este proceso de investigación se centró en un sujeto real, como estudio de caso único y análisis documentales para fundir observaciones con las aportadas por los otros.

En este estudio se propuso una metodología paradigmática que resultó una herramienta eficaz para la generación y adquisición de conjeturas, razonamientos e hipótesis, y para registrar las experiencias obtenidas con el fin de lograr los objetivos propuestos en este estudio. El estudio se convirtió en el espacio de indagación y reflexión donde se trataron de armar un rompecabezas para exponerlo frente a la crítica pública.

Como método para la recogida de datos se aplicó la observación sistemática y como investigadores mantuvimos una distancia con la propia realidad que se estaba investigando. Fueron objetivos separados los hechos observados de las ideas previas, validando así las conclusiones obtenidas. Adoptamos una posición neutral, es decir, conservamos una distancia entre el objeto y sujeto de conocimiento. Esta investigación no pretendió examinar conductas, sino interpretar sus significados para relacionarlos con la cognición. La metodología propuesta tomó como enfoque inductivo el estudio de 
un caso específico de manera detallada, es decir, se basó en factores universales determinados.

\section{Instrumentos}

La evaluación del caso se desarrolló durante 4 meses en los que se realizaron 30 sesiones de dramatización, utilizando los siguientes instrumentos para cumplir el objetivo:

1. Entrevista para niños y progenitores de la guía de intervención clínica infantil del Centro de Psicología Aplicada de la Universidad Autónoma de Madrid (UAM).

2. Registros de observación.

Se utilizó el Modelo de entrevista reseñado recabando la siguiente información: datos personales del caso, antecedentes familiares, descripción del niño, historia clínica, historia escolar, historia prenatal y perinatal, ambiente familiar, relaciones de la figuras parentales con el caso.

El método de observación utilizado fue la observación sistematizada o controlada. Mediante dicha observación sistemática pudimos cuantificar las conductas disruptivas que presentaba el caso durante las sesiones de dramatización. En contrapartida al método de observación natural, la observación sistemática es un método más riguroso, exhaustivo y preciso. Ha de partir de una hipótesis previa para ir anotando las variaciones observadas, ya sean de frecuencia entre un máximo y un mínimo o la medida del tiempo de reacción del sujeto observado ante un estímulo, la intensidad y duración en el tiempo de esa reacción.

Los datos a recoger fueron establecidos con anterioridad y definidos con un estudio previo. Los registros reflejaron de forma nítida la información que nos ofreció la actitud y conducta del caso, para evitar la pérdida de información, los datos que seleccionamos fueron de rápida detección y anotación. La toma de datos se centró en registros de acciones o actitudes disruptivas que mostró el caso a lo largo de la intervención como: negación, rabieta, riña, pelea, negativa, reto a la autoridad...

Se preparó de antemano las condiciones y situaciones que han de darse para una correcta investigación, evitando así posibles interferencias. Consideramos la importancia de no deformar la observación ni de sesgar la información importante o relevante.

La observación fue interna o participativa y activa, obteniéndose los datos desde dentro, con contacto directo con el sujeto observado, interactuando con él, en algunas situaciones intercambiando los roles. El observado, al familiarizarse con el observador, no modificó sus actuaciones, actuó con normalidad y la distorsión por tanto fue minimizada, por tanto fue de gran importancia crear esa confianza, siendo del agrado del sujeto observado, creando una posición neutra. La observación fue planificada y controlada. Como observadores consideramos las conductas sin intromisiones, tal y como son recibidas, sin introducir modificación alguna.

La secuencia del método observacional que se aplicó la llevamos a cabo según Fraisse (1970), partiendo de una primera observación en la entrevista preliminar y de los datos que nos facilitó la familia y el centro, formulando así el problema a observar: la conducta disruptiva del caso. A partir de ese problema localizado establecimos la hipótesis de como la dramatización como recurso terapéutico mejora el estado 
psicosocial y en base a esta hipótesis preparamos y realizamos la recogida de datos y su registro para finalmente concluir en base al análisis de los datos.

Se observaron las reacciones ante los estímulos que emergían de los juegos de dramatización y se tomaron los datos según el tipo de respuesta. Estas pueden ser lingüísticas o extralingüísticas, no verbales o conductas en el espacio. Así la comunicación no verbal nos ofreció información referente del nivel de ansiedad o estrés acumulado en el caso, incomodidad, nerviosismo... Se observaron los movimientos corporales, las expresiones faciales o la mirada del sujeto observado. Las posturas corporales pueden revelar desafío u oposición. Se prestó especial atención al tono de voz, la intensidad y el timbre del mismo, la interrupción al interlocutor o los silencios. Se evaluó la conducta espacial del caso, es decir, vacilaciones, situaciones en las que el sujeto muestra cercanía con el interlocutor o por el contrario se separa guardando las distancias.

Otra estrategia de recogida de información que se aplica en la investigación es el cuestionario de respuestas abiertas destinado a la familia. La información recopilada a través del cuestionario de la familia nos aportó información relevante ya que la familia pudo mantener una observación directa del estado físico y emocional del caso antes y después de la intervención de Teatro de Sombras.

\section{Procedimiento}

Contactamos con un centro externo de la Comunidad de Madrid de atención terapéutica con la finalidad de comunicar el objetivo de la investigación así como de informar de los siguientes beneficios que ofrece la dramatización a nivel psicosocial:

- Favorece las interacciones con sus iguales.

- Disminuye la labilidad emocional y la comunicación funcional.

- Ayuda a construir la identidad, potenciando los aspectos más sanos y las habilidades de cada sujeto.

- Ayuda al desarrollo del juego simbólico.

- Aumentar la gama de intereses.

- Reduce conductas oposicionistas, negativas y disruptivas.

- Mejora las habilidades sociales básicas.

- Ayuda a adquirir herramientas para una mayor auto regulación emocional

- Reduce niveles de ansiedad

- Estimula la conciencia corporal

- Refuerza la autoestima.

- Mejora el auto concepto

- Favorece la comprensión, el control y expresión de las emociones.

Posteriormente, el centro consideró nueve casos que se podían beneficiar de la intervención ya que presentaban dificultades en su construcción de la imagen corporal, una baja autoestima y dificultades en las relaciones con sus iguales. Una vez que el centro informó a las familias de los casos de los beneficios que la intervención ofrecía, contactamos con las familias con el objetivo de recibir sus consentimientos para que los casos pudieran ser entrevistados e incluidos en la intervención. A continuación, tras recibir la aprobación del centro y de las familias, procedimos a las entrevistas con los casos y las Entrevistas Generales para Niños Formato Padres. Tras la realización de las entrevistas se procedió a la selección del caso para llevar a cabo este estudio, seleccionamos al caso que más encajaba en el objetivo de la investigación, basándonos en los antecedentes, la información facilitada por el centro y las familias y las entrevistas con los casos. Una vez seleccionado el caso a observar, el centro 
facilitó un lugar físico adecuado para llevar a cabo la intervención con los nueves casos seleccionados. Esta investigación busca la mejora individual pero siempre dentro de un determinado contexto y entorno predeterminado y controlado en el que las interacciones y acciones, tanto del profesional como entre los participantes y las reacciones ante estas interacciones influyen, sin duda, en la mejora individual del caso.

Teniendo en cuenta que los juegos de dramatización liberan los cuerpos a través del movimiento y que ofrecen la posibilidad de expresar de manera creativa sentimientos, consideramos oportuno intervenir en el caso mediante juegos de dramatización. Wallon (1987), plantea como la conciencia se desarrolla en relación a aspectos sociales y biológicos y en sintonía con la evolución psicológica del niño que comprende "la emoción, el otro, el medio (físico-químico, biológico y social) y el movimiento (acción y actividad) (pp 103-132).

La dramatización, fue la vía terapéutica que utilizamos para que el caso, vivenciando su propio cuerpo, evocara imágenes del inconsciente haciéndolas conscientes. Con la finalidad de mejorar el estado psicosocial del caso y mejorar su conducta disruptiva, se llevaron a cabo 30 intervenciones de dramatización, cada sesión se desarrolló durante cincuenta minutos tres días a la semana. Durante las sesiones se aplicaron los siguientes procedimientos:

- Experimentación con el cuerpo para hacer uso de sus recursos expresivos.

- Expresión de sentimientos mediante la expresión corporal y el movimiento.

- Impulso de la fantasía e imaginación mediante la acción creativa

- Identificación de sensaciones, sentimientos y conductas propias y de los compañeros a través de juegos de dramatización.

- Comprensión de los mensajes expresado por el cuerpo.

- Uso de la expresión corporal como canalizador de emociones.

- Expresión de los miedos a través de la dramatización.

Mediante la intervención de dramatización comprobamos el efecto terapéutico de la dramatización, un elemento terapéutico alternativo a otros tipo de terapias más invasivas que ofrece un espacio dónde explorar sensaciones. El caso según se fue adentrando en los juegos de dramatización, fue encontrando un lugar donde construirse según fue experimentando con su cuerpo. Mediante la dramatización, el caso fue adquiriendo conciencia de su cuerpo, lo fue descubriendo, al teatralizar la realidad, estructuró su relación con todo aquello que le rodea para reafirmar su identidad.

Con el progreso de las sesiones de dramatización, el caso fue aumentando el tiempo de inmersión en los juegos de dramatización, según fue experimentando con su cuerpo y estableciendo con él un dialogo sensitivo e introspectivo se fueron rebajando sus niveles de ansiedad y disminuyendo sus conductas disruptivas. A través de la dramatización el caso fue exteriorizando sentimientos que sin la ayuda de la dramatización no se sentiría capaz de expresar. La dramatización es por tanto una experiencia que implica a todos los sentidos, generando una descarga de imágenes que el cuerpo proyecta. La dramatización implica un estado de introspección mediante el cual el caso fue capaz de organizar y reestructurar sus ideas de forma inconsciente, encontrándose cara a cara con sus conflictos emocionales, enfrentándose a ellos, lo que tuvo un efecto terapéutico, y colaboró en la mejora del caso, que es el fin de este estudio. 


\section{Recogida y análisis de los datos}

Durante las 30 sesiones programadas se procedió a la recogida de datos, pudimos cuantificar los comportamientos o conductas que se consideran como disruptivos, entre los que podemos destacar cólera, mal comportamiento con otros iguales, negación a determinadas órdenes, desafío o discusión con adultos, acusar a terceros, ausencia de contestación ante preguntas dirigidas directamente a él, actos rencorosos o vengativos, malas caras ante las indicaciones de los adultos, entre otros. La siguiente gráfica muestra las conductas disruptivas registradas durante las 30 sesiones de dramatización:

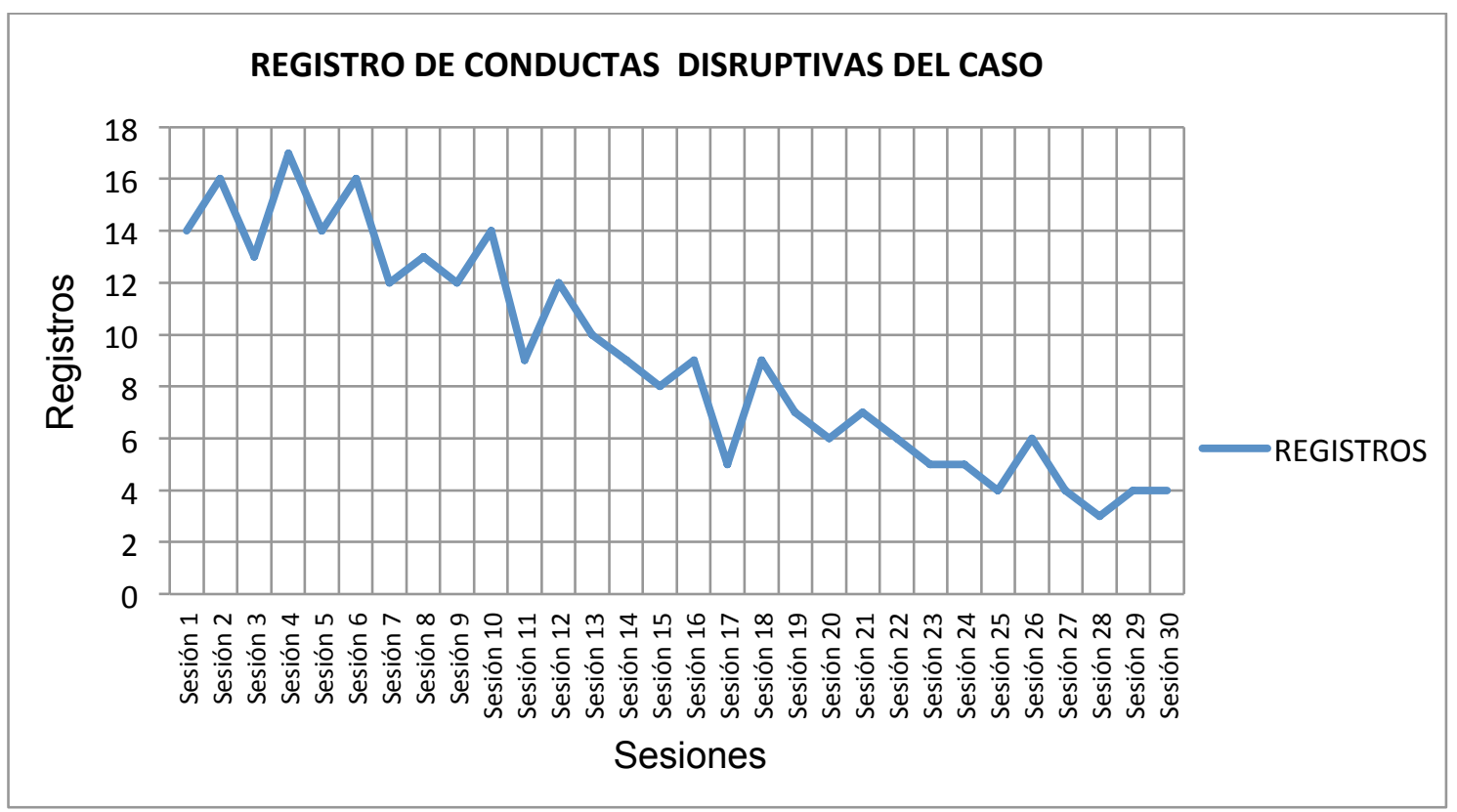

Figura 1. Registro de conductas disruptivas del caso

La gráfica muestra una disminución de conductas disruptivas del caso a lo largo de la intervención. En las primeras sesiones se observan mayor número de conductas disruptivas en relación a las últimas sesiones. Mediante el cuestionario destinado a la familia, pudimos recopilar información del caso en relación a la intervención de dramatización. La familia, afirma que el caso acudía contento a las sesiones de dramatización. Comenta que al comienzo de las sesiones se mostraba eufórico y muy contento. A la salida de las sesiones la familia valora el estado de ánimo del caso observando que se muestra excitado, cómplice con sus compañeros ya que observan que le gusta pasar tiempo con ellos. En el cuestionario se le pide a la familia que describan las dificultades más notables que muestra el caso habitualmente, describen las siguientes dificultades: muestra dificultades para respetar las normas $y$ en las relaciones con sus iguales, le cuesta mantener una conversación y mantener amistades. Se les pide que con respecto a las dificultades anteriormente señaladas $u$ otras, indiquen si han observado algún cambio tras las sesiones de dramatización. La familia confirma que el niño sale con muchas ganas de relacionarse con otros niños. Le preguntamos a la familia a través del cuestionario si los cambios observados han perdurado en el tiempo, la familia asegura que los cambios que han señalado anteriormente han permanecido en el tiempo. En las observaciones comentan que estarían dispuestos a seguir con la intervención de dramatización ya que consideran que beneficia el estado psicosocial del caso. 


\section{DISCUSIÓN}

Teniendo en cuenta los datos registrados, podemos confirmar la hipótesis de como la dramatización como recurso terapéutico resulta eficaz para la mejora psicosocial de menores que presentan un comportamiento disruptivo. A través de la expresión artística y corporal que implica la dramatización es posible reducir los niveles de ansiedad del niño/a reduciendo así sus conductas disruptivas. Hemos podido registrar datos que muestran como durante el progreso de la intervención de dramatización han ido disminuyendo las conductas disruptivas del caso y a través de la observación sistemática hemos contemplado como los tiempos de inmersión del caso en los juegos de dramatización han ido aumentando, lo cual evidencia que existe una relación que podemos considerar como significativa entre la emoción que evoca la dramatización, los niveles de ansiedad del caso y sus conductas disruptivas. Damasio (1994) ya apuntaba la importancia de la emoción en el desarrollo del niño/a exponiendo como la emoción le permite adquirir la "sensación de sí mismo". La experiencia vivenciada mediante la dramatización constituye una experiencia significativa que deposita ciertas huellas fisiológicas en el cuerpo y le permite al niño/a adquirir dicha "sensación de sí mismo".

Veo la esencia de la emoción como el conjunto de cambios en el estado corporal que son inducidos en multitud de órganos por los terminales de las neuronas, baja el control de un sistema cerebral dedicado, que está respondiendo al contenido de pensamientos en relación a una entidad o acontecimientos determinados. (Damasio, 2011, p. 199)

Los resultados del estudio experimental prospectivo de caso único ponen de manifiesto como las conductas disruptivas del caso son susceptibles de aminorar a través de la intervención de dramatización y favorece el estado psicosocial del caso. Los resultados obtenidos van en la línea de los últimos aportes en el campo de la neurología que nos hablan de la plasticidad neuronal que demuestra que la experiencia deja una huella en la red neuronal capaz de modificar conductas o comportamientos como ya anunciaban Ansermet, F y Magistretti en el año 2006.

El fin de esta investigación es evidenciar los beneficios que el Arteterapia ofrece a menores que son incluidos en los programas de necesidades educativas especiales para que puedan aplicarse a otras situaciones, es decir, transformar la teoría en un conjunto general de ideas que orienten la acción. Desde esta perspectiva, este estudio significa un paso más, que se espera que abra nuevos caminos. La presentación de este caso puede suponer el punto de arranque de nuevas investigaciones 0 proporcionar un eje vertebrador que facilite la gestión de producción de nuevos recursos educativos y terapéuticos.

\section{Referencias}

Alvarez, H \& Ollendick, T (2003). Individual and psychosocial risk factors. En C.A. Essau (Ed.): Conduct and oppositional defi ant disorders: Epidemiology, risk factors, and treatment (pp. 97-116). Mahwah, NJ: Lawrence Erlbaum Associates Publishers.

Ansermet, F \& Magistretti, P. (2006). A cada cual su cerebro. Buenos Aires: Katz.
Armas, M. (2007). Prevención e Intervención ante problemas de conducta. Estrategias para centros educativos y familias. Madrid: Wolters Kluwer España.

Aristóteles (1990). Poética. Caracas: Monte Ávila editores (Traducción del griego, introducción y notas de A.J Cappelletti).

Blatner, A. (1988). Foundations of Psychodrama. History, Theory and Practice.(Third Edition). New York: Springer Publishing Company. 
CIE 10. (1994). Trastornos mentales y del comportamiento. Madrid: Meditor.

DSM-IV (1994). Diagnostic and statistical anual of mental disorders, 41ed. APA: Washington: D.C.

Emunah, R. (1994). Acting for Real: Drama Therapy, Process, Technique and Performance. Levittown, PA: Brunner Mazel.

Fraisse, P. (1970) Le méthode experimentale. En P. Fraisse y J. Piaget (eds), Traitté Psychologie Experimnetales Y: Historie et Méthode. París: P.U.F

Carla Morales Pillado, Manuel García Tabuyo, Carolina Álvarez Ortiz, Marta Gervás Sanz, Rebeca Pardo Cebrián, Óscar Pérez Cabrero, Marta de la Torre Martí y Marta Santacreu Ivars (2015) Guía de intervención clínica infantil. Centro de psicología aplicada. Universidad Autónoma de Madrid.

Lacan, J. (1946). El Estadio del espejo en Escritos I. Buenos Aires, Siglo Veintiuno Editores

Lapierre, A.; Aucouturier, B. (1980): El cuerpo y el inconsciente en educación y terapia. Barcelona. Editorial Científico-médica.

Ley Orgánica 2/2006, de 3 de mayo, de Educación. Publicado en: « $\mathrm{BOE}$ » núm. 106.

McMillan, J.H. \& Schumacher, S. (2001) Research in education: A conceptual introduction ( $5^{\mathrm{a}}$ ed.) New York: Addison Wesley Longman.

Moreno, J. (1946). Psychodrama. First Volume. (Cuarta edición: 1972). Ambler, PA (1985): Beacon House. Mora.

Rigau-Ratera, E., García-Nonell, C. \& Artigas-Pallarés, J. (2006). Tratamiento del trastorno oposicionista desafiante. Revista de Neurología, 42(2): 83-88.

Stake, R. (1985). Case Study. En Nisbet, J. (Ed) World Yearbook of Education 1985. Research, Policy and Practice. London: Kogan Page.

UNESCO, (2011), Revision of the International Standard Classification of Education (ISCED).

Wallon, H. (1987). Psicología y educación del niño. Una comprensión dialéctica del desarrollo y la Educación Infantil. Madrid: Visor-Mec. 